RF-Synchronized Imaging for Particle and Photon Beam Characterizations*

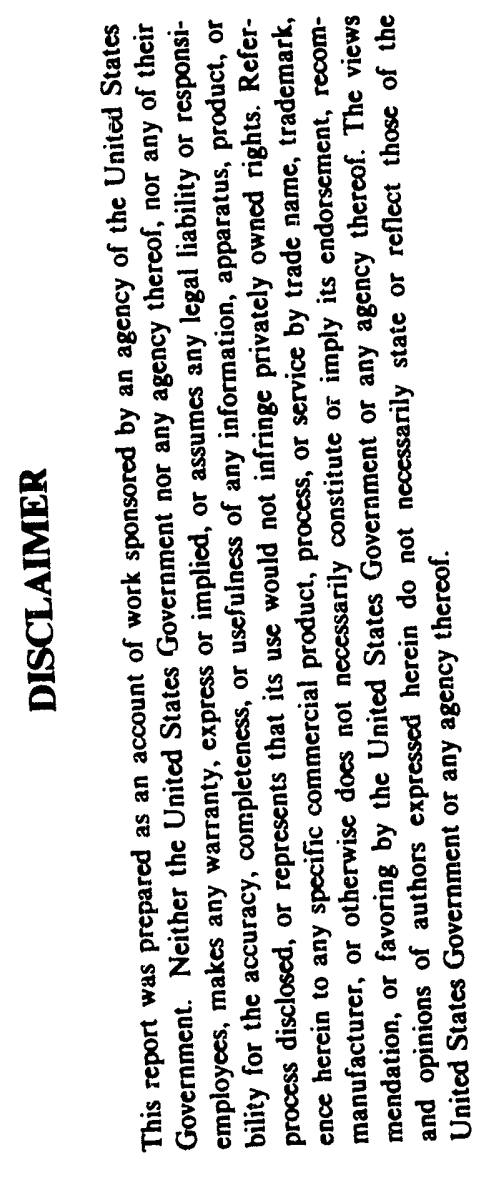

\author{
by \\ A. H. Lumpkin \\ Advanced Photon Source \\ Accelerator Systems Division \\ Argonne National Laboratory \\ 9700 S. Cass Avenue - Bldg. 362 \\ Argonne, Mlinois 60439 USA \\ Telephone: (708)252-4879 \\ FAX: (708)252-7187
}

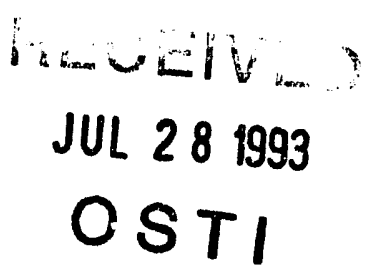

*Work supported by the U.S. Department of Energy, Office of Basic Energy Sciences, under Contract No. W-?1-109-ENG-38.

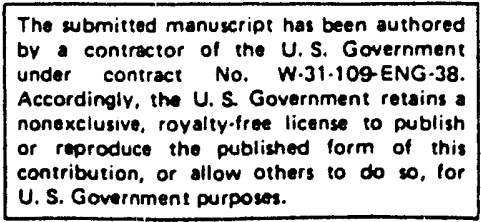




\title{
RF-Synchronized Imaging for Particle and Photon Beam Characterizations*
}

\author{
A. H. Lumpkin \\ Advanced Photon Source \\ Argonne National Laboratory \\ 9700 S. Cass Avenue, Argonne, IL 60439
}

Abstract

The usefulness of imaging electro-optics for $\mathrm{rf}$-driven accelerators can be enhanced by synchronizing the instruments to the system fundamental frequency or an appropriate subharmonic. This step allows one to obtain micropulse bunch length and phase during a series of linac bunches or storage ring passes. Several examples now exist of the use of synchroscan and dual-sweep streak cameras and/or image dissector tubes to assess micropulse scale phenomena (10 to 30 ps) during linac and storage ring operations in the U.S., Japan, and Europe. As space permits, selections will be presented from the list of phase stability phenomena on photoelectric injectors, micropulse bunch length during a macropulse, micropulse elongation effects, transverse Wakefield effects within a micropulse, and submicropulse phenomena on a stored beam. Potential applications to the subsystems of the Advanced Photon Source (APS) will be briefly addressed.

\section{INTRODUCTION}

Significant advantages for imaging instrumentation used for diagnostics and experiments on rf-driven accelerators can be realized if the electro-optics are synchronized to the accelerator fundamental frequency or a subharmonic of the master oscillator. This step allows one to obtain bunch length and phase during a series of linac beam bunches or storage ring turns. Over the last few years synchroscan and dualsweep techniques using streak cameras [1-3] and/or image dissector tubes [4] have been used to assess micropulse scale phenomena (10 to $30 \mathrm{ps)} \mathrm{during} \mathrm{linac} \mathrm{and} \mathrm{storage} \mathrm{ring}$ operations in the U.S., Japan, and Europe. Space constraints will limit discussion to selections from the list of phase stability phenomena on a photoelectric injector, micropulse bunch length variation during a macropulse, micropulse elongation effects, transverse Wakefield effects within a linac micropulse, and head-to-tail bunch instabilities in a stored beam. Potential applications to the subsystems of the Advanced Photon Source (APS) will be addressed. Although the number of $\epsilon$ xamples is not large, the applications described cover the gamut of if-accelerators one might encounter within this conference's purview.

*Work supported by the U.S. Department of Energy, Office of Basic Energy Sciences, under Contract No.W-31-109-ENG-38.

\section{BACKGROUND AND PROCEDURES}

In some of the earlier attempts on using single-shot streak cameras to assess the micropulse bunch length of beams from a variety of linac configurations, one encountered triggering jitter from the standard pulse generators at that time as well as the internal streak camera timing jitter. The first area we solved by using a synchronous delay unit developed at EG\&G (SBO) that referenced itself to the $108.3-\mathrm{MHz}$ subharmonic and then allowed time delays with low jitter for triggering the streak camera. Basically at that time, we dropped the \pm 100 ps jitter to about \pm 20 ps [5]. Practically speaking, this meant the streak image stayed localized in the 400-ps field-of-view instead of bouncing up and down by one fourth the viewed scene on the time axis. Within the freeelectron-laser (FEL) community where I was working at that time, the need for phase stability of the e-beam relative to the oscillator cavity caused us to look at a tradeoff. As seen in Table 1 (Hamamatsu C1587 mainframe), one can significantly

Table 1 Summary of Streak Camera Module Properties

\begin{tabular}{cccc} 
Module: & $\begin{array}{c}\text { Temporal } \\
\text { Resolutlon } \\
\text { (FWH, ps) }\end{array}$ & $\begin{array}{c}\text { Phase Jllter } \\
\text { (FWHM, ps) }\end{array}$ & $\begin{array}{c}\text { Phase Jitter } \\
\text { (rms, ps) }\end{array}$ \\
\hline $\begin{array}{c}\text { Fast Sweep } \\
\text { (single) }\end{array}$ & $<2$ & $10-15$ & $4-6$ \\
$\begin{array}{c}\text { Synchroscan } \\
\text { (M 1954) }\end{array}$ & $6-8$ & 4 & 1.7 \\
$\begin{array}{c}\text { Synchroscan } \\
\text { (M 1954-10) }\end{array}$ & $-3-4$ & 2 & $\sim 0.8$
\end{tabular}

reduce the streak camera internal jitter by using the synchroscan mode. In this mode, the $108.3-\mathrm{MHz}$ if signal is injected directly into the camera's sweep circuitry so the plates are driven at the of frequency and with much lower timing jitter [2]. The temporal resolution was reasonable in the Hamamatsu M1954 system (8-10 ps), but was improved in the M1954-10 unit with 3-4 ps (FWHM) resolution and an ms jitter $<1$ ps.

Additionally, once in the synchroscan mode, one could synchronously sum the signals from a series of micropulses (such as from optical transition radiation (OTR)) in a linac or turns in a storage ring. To obtain some knowledge of evolution within these much longer timescales, the orthogonal set of plates in the tube were given a time-dependent ramp (Fig. 1). This allows micropulse phenomena to be tracked during macropulse timescales. We identified a number of 
phenomena that previously were difficult to assess. These included observation of the time structures of the fieldemission electrons reported at the last PAC [6], and a number of transient phase phenomena in photoinjectors, linacs, and potentially in storage rings. The work here uses visible-UV imaging, but could be extended in principle to the VUV, XUV, and $x$-ray regimes. The techniques directly apply where a conversion mechanism transforms the particle beam information into the detectable photon field. Descriptions of many of these results are given in more detail elsewhere [3]. I will concentrate on data from the rf-linac driven FEL programs at Los Alamos and Boeing, which include the first dual-sweep results on a drive laser for a photoinjector and for linac-driven FELs. It should be noted that work in Japan was the first on a storage ring [1], while additional effort with a different dual sweep camera has been going on at CERN by E. Rosca in 1990-93. [7]

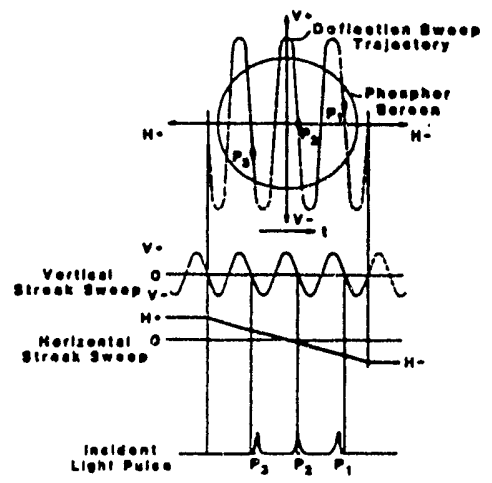

Figure 1 A schematic of the dual-sweep streak techniques.

\section{EXAMPLES OF RESULTS}

As discussed earlier, the usefulness of these techniques can be graphically demonstrated by measurements made in various stages of the production and use of a charged-particle beam in an accelerator system. Possible uses include the experimental area, a FEL wiggler, injection into a storage ring and its undulators, etc.

\section{A. Photoinjector Drive Laser}

Laser phase jitter was not identified as an issue in an early configuration of the $1-\mathrm{MeV}$ photoinjector at Los Alamos because the jitter was much less than the $\sim 60$ ps bunch length. However, phase stability issues soon became evident in the next configuration of a 6-MeV photoinjector, followed by a second $12-\mathrm{MeV}$ accelerator [8]. In these operations of the $1300-\mathrm{MHz}$ rf cavity and with drive laser bunch lengths of 5-15 ps, drive laser phase jitter shot-to-shot directly impacted particle beam stability shot-to-shot (macropulse-to-macropulse). Because there has been increased activity in the use of these devices, it is appropriate to remind the accelerator community of the obvious: you need a stable laser. The mode-locked $\mathrm{Nd}$ :YLF oscillator configuration at Los Alamos is shown in Fig. 2. Although details will not be presented here of its operation, severul references exist [9]. We actually looked at both the fundamental, $\lambda=1.053 \mu \mathrm{m}$, before and after the pulse compressor as well as the final doubled-frequency component after the KTP crystal which was delivered to the photocathode.

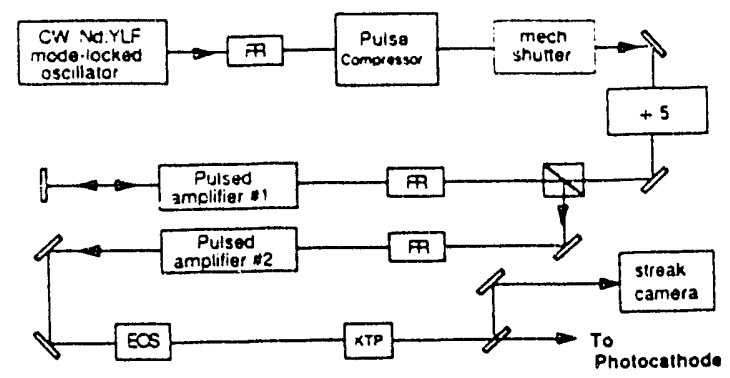

FR Faraday rotator optical isolator

EOS - Electro-ootic shutter

KTP. Polassium titanyl phosphate trequecy doubling crystal - 5 . F ast pockeis cell snuner !ransmitting every !inh puise

Figure 2 Schematic of modelocked Nd:YLF drive laser on the Los Alamos photoinjector.

The initial data that we saw were synchroscan streak images on the video monitor that moved (jittered) on the time axis more than the pulse length. There, of course, was a discussion over whether this image jitter was due to streak camera timing jitter or laser jitter. We resolved this by playing back from the encoded video record into the digitizer a series of consecutive macropulses and displaying them in sets of four. In this case (Fig. 3), the horizontal axis is tle time axis, and the motion of the center of the profile is evident (macropulse integrated). From the same set of video-encoded
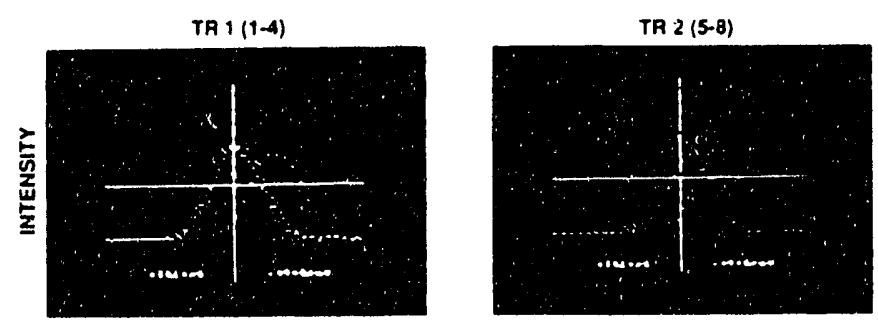

Figure 3 Drive laser micropulse temporal profiles for 8 consecutive macropulses taken using the synchroscan streak camera.

data we analyzed the electron spectrometer images at the end of the linac for the same 16 macropulses and tracked the ebeam energy. As seen in Fig. 4, a strong correlation is observed between the relative drive laser phase jitter and the e-beam energy. A sensitivity of about $0.1 \% \Delta \mathrm{E} / \mathrm{ps}$ at $17 \mathrm{MeV}$ was observed which was in reasonable agreement with PARMELA simulations. $[10,11]$ This was cross-checked against a deliberate change of a NARDA phase shifter on the drive laser where a 14-ps shift resulted in a $1.5 \%$ energy change. In fact, in our limited field-of-view of the energy in the spectrometer, for the larger phase jitters ( $>5 \mathrm{ps}$ ) the energy shifted out of view! Further corroboration of the phase jitter in the drive laser was seen when an active Lightwave phase 
feedback unit was incorporated into the laser system, and these effects were significantly reduced as shown in Fig. 32 of Ref. 3.

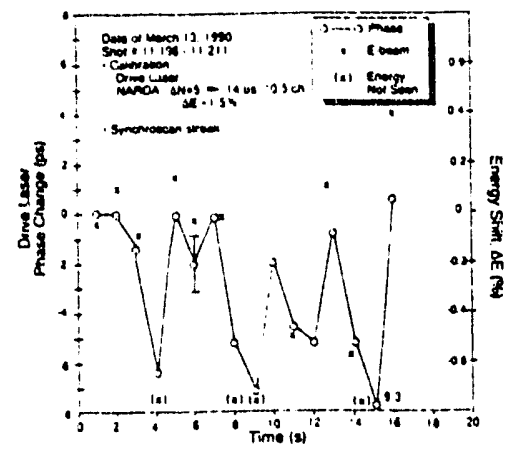

Figure 4 Comparison of observed drive lasesr phase jitter and e-beam energy for the same 16 macropulses.

This was a shot-to-shot (intermacropulse) phenomenon, but there were additional intra- or submacropulse effects in the

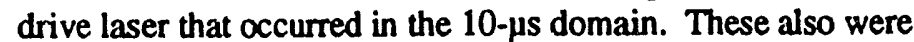
sufficient to affect FEL operations within a macropulse. In this case, the dual sweep feature of the camera was used. Eight time-samples were selected for analysis across the nominal 20- $\mu$ s-long macropulse. Both single and 20macropulse averages were used. The nominal bunch length was still 7-10 ps. In Fig. 5, for some data (File 9) the phase

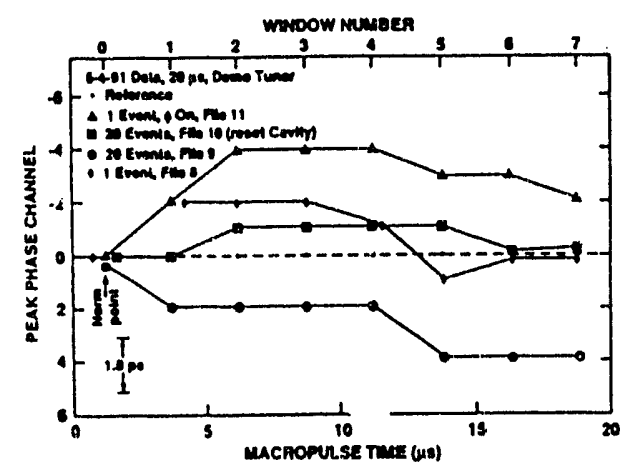

Figure 5 Submacropulse phase slew effects in the drive laser 20- $\mu$ s long macropulse.

of the laser drifted about $4 \mathrm{ps}$ (which would be $0.4 \% \Delta \mathrm{E}$ at 17 $\mathrm{MeV}$ and $0.8 \% \Delta \lambda$ at $10 \mu \mathrm{m}$ ) during the macropulse. Resetting the cavity (File 10) resulted in stable behavior within the error of the measurement $(\sim 1 \mathrm{ps})$, as referenced to the normalization line. More details are in Ref. 11.

\section{B. Photoinjected Linac}

Just as the tining (relative to the $\mathrm{rf}$ field) of the release of the electrons from the photocathode may be expected to cause an energy effect, it also is related to transit time through the photoelectric injector (PED) accelerator first cell and the rf bunching across the micropulse available from the field gaadient. Figure 6 shows our measurement at $17 \mathrm{MeV}$ on the observed bunch length and transit time. In the dual images we "simultaneously" detected (1) a split-off fraction of the drive laser light beam just before the photocathode and (2) the subsequent electrons at $17 \mathrm{MeV}$ via a Cherenkov converter. Figure 7 shows the variation of this phenomenon as a function of drive laser phase. The elongation due to space change forces increases as the effective field gradient is reduced. The PARMELA simulation by B. Carlsten (solid line) is in good agreement [8].

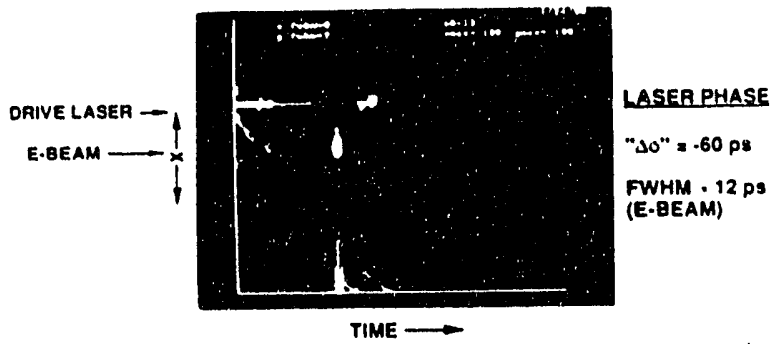

Figure 6 Streak images of the drive laser and e-beam micropulses for one laser phase setting.

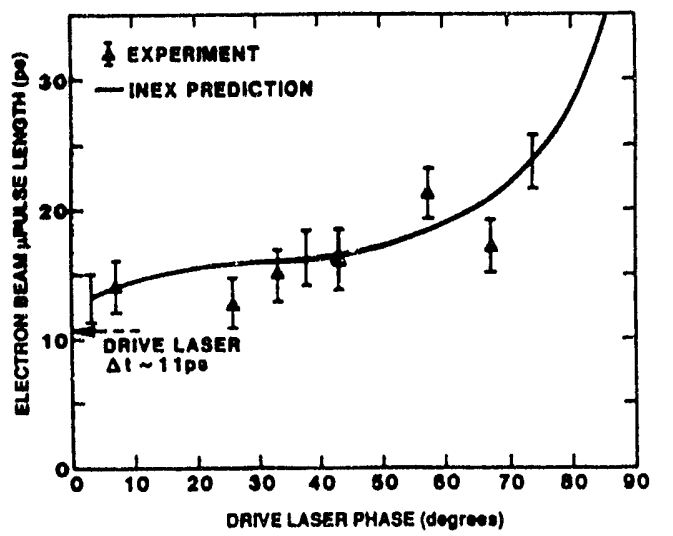

Figure 7 Plot of observed e-beam bunch length versus drive laser relative phase. The drive laser bunch length was monitored at $11 \mathrm{ps}$.

\section{110-MeV Linac}

The next example is from operations a few years ago on the $110-\mathrm{MeV}$ linac injected with electrons from a thermionicgun with of buncher system (at Boeing in Seattle, WA). This linac beam was used to drive a visible FEL oscillator experiment. The output spontaneous emission or lasing from the 5-m wiggler was optically transported to a streak/spectrometer system [12]. During the course of the various configurations of the experiment including a ringresonator mode, the electron-beam bunch length was shown to vary during the macropulse. As shown in Figs. 8 and 9, the bunch length FWHM started at $\sim 25$ ps and was reduced to $\sim 10$ ps (as was its intensity) by the end of the 100- $\mu$ s-long macropulse. Since FEL gain is strongly dependent on peak current, inefficient operations resulted. The e-beam problem was traced to the combination of an if phase slew, an energy slew, and a nonachromatic bend in the beam transport. 


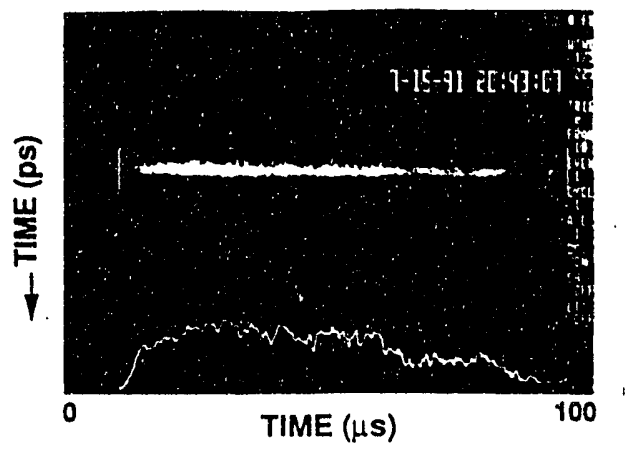

Figure 8 Dual-streak image of the e-beam micropulse bunch length variation during the $100-\mu$ s long macropulse.

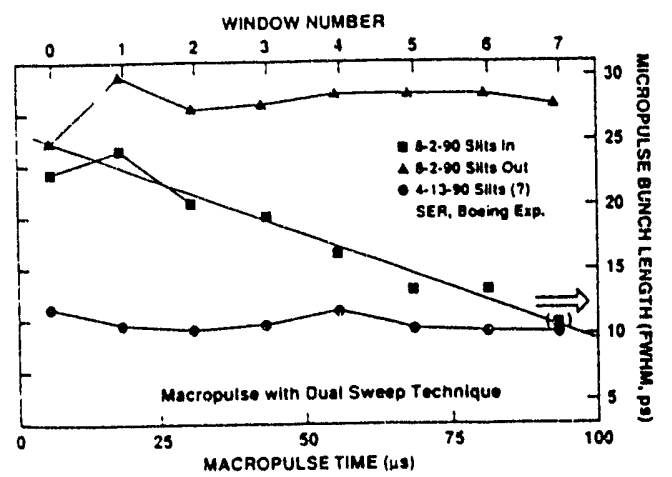

Figure 9 Eight-window samples analyzed on the macropulse time axis from Fig. 8 showing the 25- to 10-ps bunch length variation in the 8-2-90 data (squares).

Another example of dual-sweep data is shown in Fig. 10 involving the FEL output. The laser intensity was strongly modulated during the macropulse while we simultaneously measured the 28 -ps (FWHM) lasing micropulse. The modulation was correlated with an e-beam energy centroid modulation measurement (using a stripline BPM in a dispersive location) arid traced to the rf system (the pulseforming network). When the if system was corrected, significant improvement in e-beam parameter stability and FEL quality resulted [13].

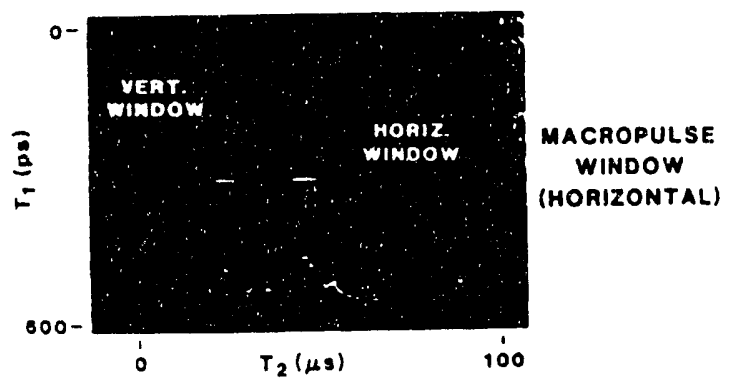

Figure 10 Dual-sweep streak image of the Boeing visible FEL output showing intensity modulation during the macropulse.

\section{Transverse Wakefields}

In most applications for low-emittance beams, it is not sufficient to generate them: you must preserve the beam as you transport it. As an example, off-center transport through subsequent linac tanks can degrade the beaun. An example is shown from the 40-MeV linac at Los Alamos [14]. Figure 11 shows the experimental setup where a synchroscan streak camera was used to view the forward lobes of the optical transition radiation (OTR) foil at station \#4 after the fourth tank (D). Another OTR screen after Tank $C$ was used to determine the position entering Tank $D$. Steering magnets were used to change the beam offset as it went through Accel. $D$ to screen 4. In Fig. 12, for $Q=5 n C$ per micropulse, the time-resolved $\mathrm{x}$-profile is shown for different steering/offset conditions. The images are the synchronous sum of the micropulses in one macropulse, and one can see that the direction of the head-to-tail kick changes on the micropulse time scale as the beam is steered differently through Tank D. In fact, Fig. 12 a,b shows one can actually compensate for the transverse kick and reduce the observed time-averaged spatial $x$-profile FWHM from $0.8 \mathrm{~mm}$ to $0.4 \mathrm{~mm}$. More detailed discussion is given elsewhere in this conference [15].

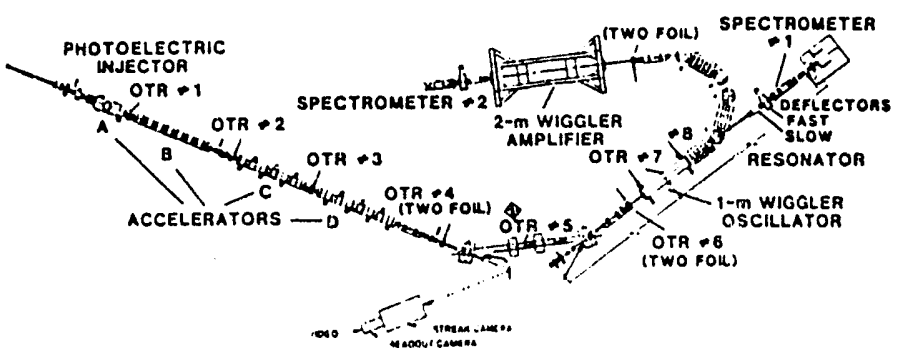

Figure 11 Experimental setup for the synchroscan streak camera on the Los Alamos 40-MeV linac. The forward OTR radiation from the screen after Tank $D$ is detected.

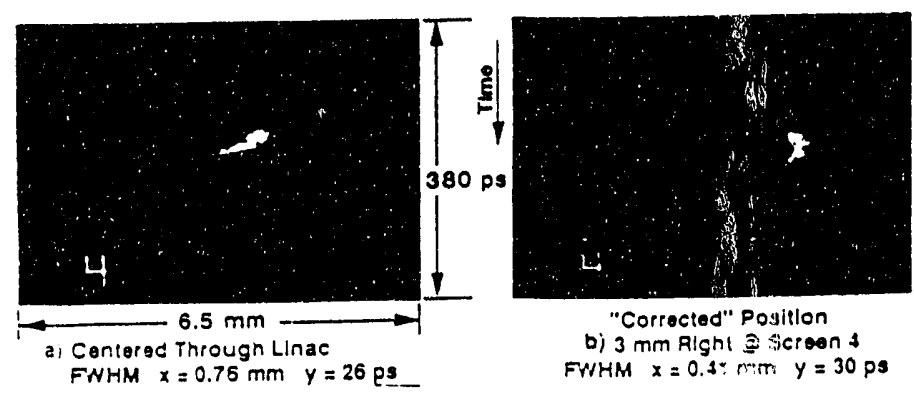

Figure 12 Synchronous sum of submicropulse sercak data exhibiting "head-to-tail" kick changes with steering through the linac.

Similar phenomena have been detected at SLAC and more recently at LEP by E. Rossa using a dual-sweep streak camera. Figure 13 is a reproduction from the CERN work illustrating the head-to-tail transverse effect in LEP [7]. The micropulse time scale is horizontal and the vertical axis is related to the transverse spatial projection. The "tilts" of some of the bunch projections imply the head-to-tail kicks varied on different turns. 


\section{ACKNOWLEDGEMENTS}

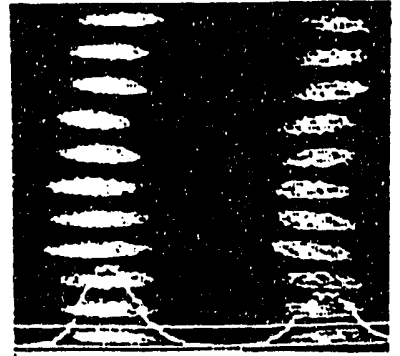

Figure 13 Reproduction of dual-sweep data from the LEP experiment by E. Rossa, et al. [7] showing head-to-tail kicks in a single bunch over 11 turns. The left set is $y-t$ (sideview) and the right set is $\mathrm{x}-\mathrm{t}$ (top) view.

\section{E. Potential Applications at APS}

At APS the undulator test line will involve a lowemittance beam in a small bore linac structure. Potential transverse Wakefield effects such as described earlier will be considered. In the $7-\mathrm{GeV}$ storage ring, we have revisited the possibility of longitudinal instabilities and how to measure them. A workshop was held on this topic in 1989 specifically for the APS storage ring [16]. L. Emery's recent simulations using the impedance file prepared for the APS storage ring indicates that in addition to bunch lengthening (about 30\% for $5 \mathrm{~mA}$ /bunch), the longitudinal phase space could vary as a function of time [17]. Variation of the projection on the time axis after injection and during damping for different currents should be directly measurable by the dual-sweep streak techniques. Additional work is needed to simulate how this phenomenon might carry over to the transverse phase-space projections.

\section{SUMMARY}

In summary, a number of diagnostic applications are noticeably enhanced by using $\mathrm{rf}$-synchronization techniques for the streak camera systems. Both synchroscan and dual-sweep features are important. The synchroscan allows the synchronous sum of pulses to be done with good temporal resolution, and it also allows the use of less bright conversion mechanisms (such as OTR) to be used in intercepting beam experiments. The dual-sweep allows the tracking of psphenomena over much longer time scales on a linac macropulse or many turns in a circular accelerator. The techniques have been applied successfully to both particle and photon beams on the submicropulse, submacropulse, or single pass mode. They have been applied to rf-linacs driving FELs and should be useful to linacs injecting damping rings or storage rings (as well as the rings). It is expected they will be applied to the subsystems of the APS, and hopefully be eventually extended to study the $x$-ray beams from the APS.
The author acknowledges the staff and technicians at the Los Alamos FEL facility (M. Wilke, S. Apgar, J. Early, D. Feldman, P. O'Shea, et al.) and the Boeing FEL facility (D. Dowell, P. Johnson, K. Davis) for their assistance during operations and experiments; the Simulation group (M. Schmitt, B. Carlsten, et al.) at Los Alamos for many useful discussions; M. Couprie of Orsay for information on the Super-ACO experiment; E. Rossa of CERN for discussions of his dualsweep system; and L. Emery of Argonne for his recent simulation of APS longitudinal phase space eifects.

\section{REFERENCES}

[1] Kazuhisa Nakajma, and Atsushi Ogata, "Variation of Longitudinal Beam Profile During Acceleration in Tristan Main Ring," 6th Symposium of Accel. Sci. and Tech., Tokyo, Japan, Oct. 27-29, 1987.

[2] Y. Tsuchiya, et al., SPIE Vol. 693 Highspeed Photography, Videography and Photonics IV, p. 125 (1986).

[3] Alex H. Lumpkin, "Advanced Time-Resolved Imaging Techniques for Electron-Beam Characterization, $A I P$, No. 229, p. 151 (1991), and references therein.

[4] M. Couprie, et al., NIM A318, p. 59 (1991).

[5] A. H. Lumpkin, et al., NIM $\underline{A} 285$, p. 17-22 (1989).

[6] Alex H. Lumpkin, et al., Proceedings of the 1991 PAC, Vol. 3, p. 1967 (1991).

[7] E. Rossa, et al., "Real-time Measurements of Bunch Instabilities in LEP in Three Dimensions Using a Streak Camera," CERN, 92-SL-92-155.

[8] A. H. Lumpkin, et al., NIM A304, p. 379-385 (1991).

[9] J. W. Early, et al., NIM A318, p. 381-388 (1992) and references therein.

[10] B. E. Carlsten (Los Alamos National Laboratory), private communication, 1990.

[11] Alex H. Lumpkin and James W. Early, NIM A318, p. 389-395 (1992).

[12] A. H. Lumpkin, et al., NIM A296, p. 169-180 (1990).

[13] D. H. Dowell, et al., NIM A318, p. 74-80 (1992).

[14] Alex H. Lumpkin and Mark D. Wilke, "Time-Resolved Electron Beam Characterizations with Optical Transition Radiation," presented at the 14th International FreeElectron Laser Conference, Kobe, Japan, Aug. 23-28, 1992.

[15] P. G. O'Shea, et al., "Measurement of Single-Bunch Transverse Wakefield Effects in an Electron Linac," these proceedings.

[16] Proceedings of the Impedance and Bunch Instability Workshop, Argonne National Laboratory, Argonne, Illinois, Oct. 31-Nov. 1, 1989, ANL/APS/TM-5 (April 1990).

[17] L. Emery (Argonne National Laboratory), private communication, May 1993. 

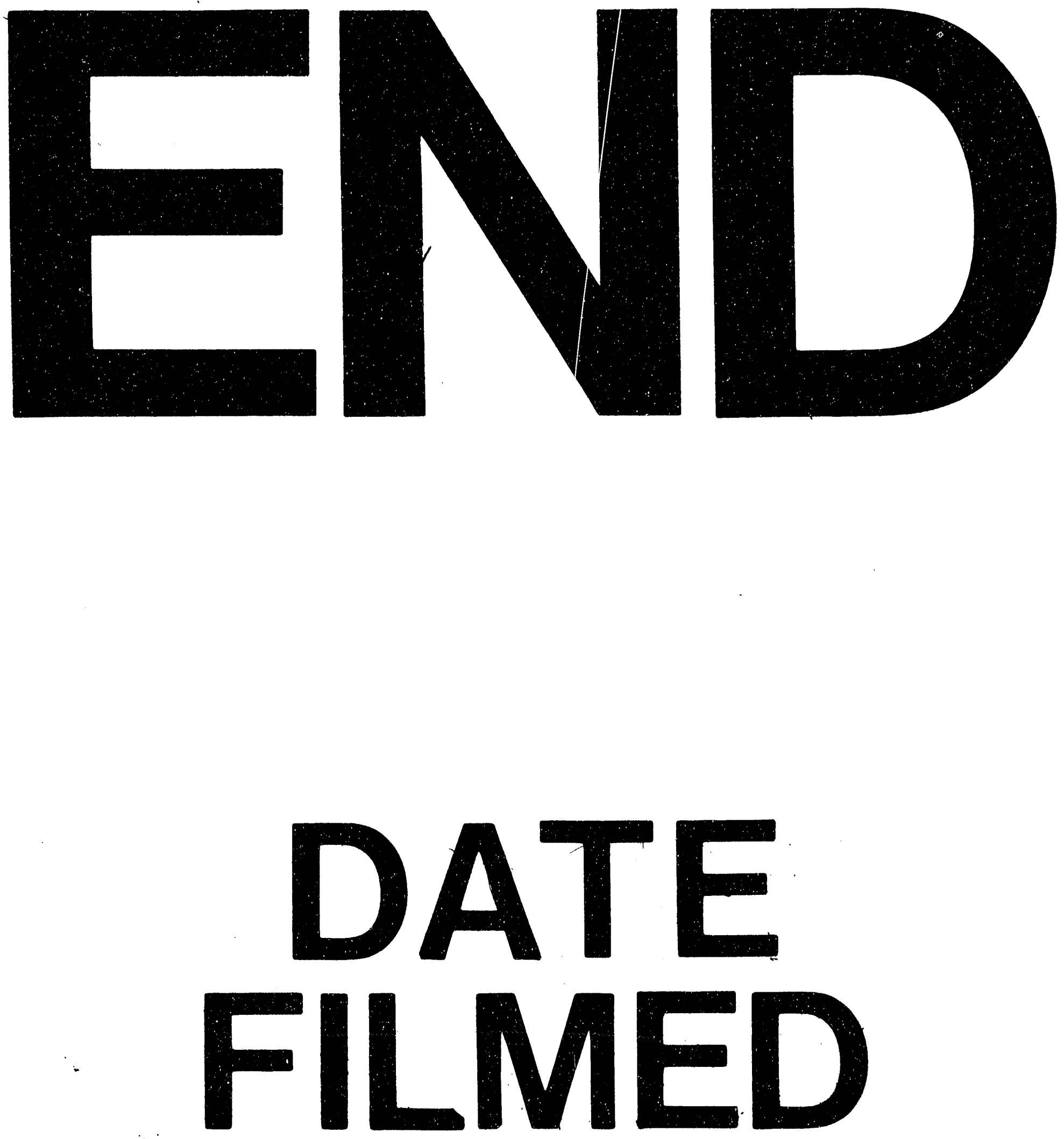

1

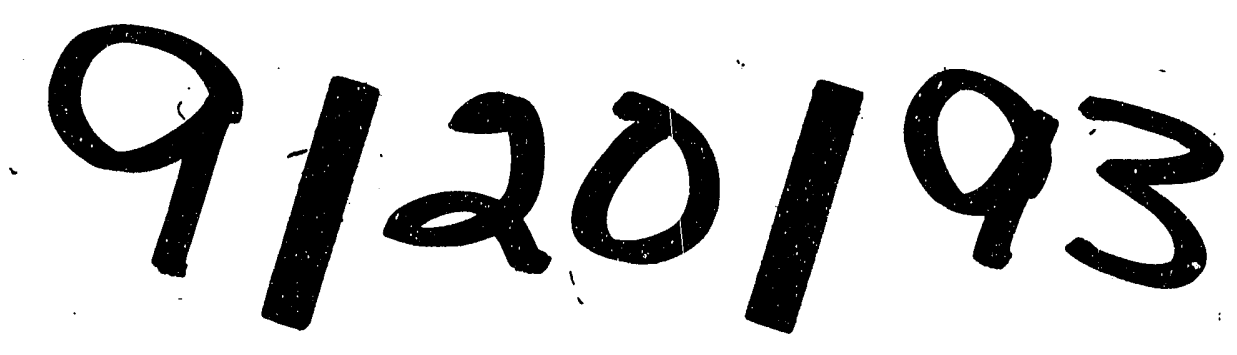


$1 \ldots$, 